\title{
La réforme éducative en Italie
}

Education reform in Italy

La reforma educativa en Italia

\section{Nicoletta Biferale}

\section{CpenEdition}

\section{Journals}

Édition électronique

URL : https://journals.openedition.org/ries/2421

DOI : 10.4000/ries.2421

ISSN : 2261-4265

Éditeur

France Education international

Édition imprimée

Date de publication : 1 décembre 2000

Pagination : 63-78

ISSN : 1254-4590

\section{Référence électronique}

Nicoletta Biferale, "La réforme éducative en Italie », Revue internationale d'éducation de Sèvres [En ligne], 28 | 2000, mis en ligne le 01 décembre 2003, consulté le 09 juillet 2021. URL : http:// journals.openedition.org/ries/2421 ; DOI : https://doi.org/10.4000/ries.2421 


\title{
La réforme éducative en Italie
}

\author{
Nicoletta Biferale
}

\section{Résumé}

Le système éducatif italien est en pleine rénovation, suite à un débat politique intense. Dès 1996, une décentralisation des structures administratives a conduit à une plus grande autonomie des établissements scolaires, concrétisée par l'obligation pour chaque centre de présenter un plan d'offre de formation (POF). Les réformes en préparation prévoient une réorganisation des cycles scolaires et de nombreuses mesures à la fois structurelles et pédagogiques, parmi lesquelles figurent en priorité le développement de l'enseignement des langues étrangères, la valorisation des nouvelles technologies de l'information et de la communication, la formation permanente des enseignants.

\section{Education reform in Italy}

Amidst widespread political debate, Italy's education system is being radically overhauled. Since 1996, administrative decentralisation has given schools greater operating autonomy along with the obligation for each centre to devise a training programme (POF). The priorities identified for reforms currently being prepared include reorganising the academic calendar, developing foreign language learning, increasing the use of new information and communications technologies and improving lifelong learning for teachers.

\section{La reforma educativa en Italia}

El sistema educativo italiano está en plena renovación, tras un debate político intenso. Una descentralización de las estructuras administrativas otorgó a partir de 1996 una mayor autonomía a los establecimientos escolares, cada centro se vio en la obligación de presentar un Plano de Oferta de Formación (POF). Las reformas en curso preven una reorganización de los ciclos escolares y numerosas medidas a la vez estructurales y pedagógicas, entre las cuales figura en prioridad la enseñanza de idiomas extranjeros, la valorización de las nuevas tecnologías de información y comunicación así como la formación permanente del cuerpo docente. 
Cet article vise à présenter les différents aspects de la réforme de l'éducation en Italie au moment où une loi, après plusieurs années de débat au niveau politique et éducatif, dessine le cadre législatif du futur aménagement du système scolaire du pays.

La première partie tentera de faire le point sur la décentralisation des structures administratives et l'entrée en vigueur de l'autonomie, qui concerne à la fois la didactique et l'organisation des établissements scolaires, condition préalable à la réforme elle-même; la deuxième partie est consacrée à l'analyse de la loi sur la réorganisation des cycles et à la présentation des travaux de la commission des Sages, chargée d'élaborer un premier document qui servira de base à la prochaine proposition de loi.

\section{Autonomie et décentralisation}

Dès le début de la Xıı législature, en mai 1996, le gouvernement s'est trouvé face à un choix à réaliser pour la rénovation du système scolaire du pays : soit une politique souple d'ajustement à travers les changements les plus urgents à opérer; soit une politique réformatrice plus radicale qui vise globalement le système, dans le but d'adapter l'instruction et la formation aux défis de la société contemporaine. L'ancien ministre G. Berlinguer a opté pour la deuxième solution et Tullio De Mauro, qui a pris sa place le 26 avril 2000, est en train d'accomplir le plan général de réforme lancé il y a quatre ans.

L'exigence ressentie en Italie par le monde politique, les spécialistes du secteur éducatif et la société en général peut se synthétiser dans la nécessité de mettre l'école au service du développement du pays, pour que l'Italie soit compétitive sur le marché international et participe en même temps au processus d'harmonisation des parcours formatifs au niveau européen. Dans le contexte d'une société complexe, en évolution constante, fondée sur l'information et la communication informatiques, obligée de s'adapter aux nouvelles stratégies économiques mondiales, émerge le besoin de bâtir une école qui tienne compte de la rapidité du changement et qui offre à tous une garantie de réussite à travers des parcours flexibles et diversifiés. Le modèle rigide du passé ne peut donc plus s'adapter ni aux changements sociaux intervenus, ni à la nécessité de fournir à tous les jeunes des instruments qui leur permettent de faire un choix conscient pour leur avenir et de s'intégrer dans un monde où la perspective économique semble être le marché global de l'emploi.

Un système scolaire rigide et verticalisé doit céder le pas à l'école de l'autonomie, respectueuse des exigences propres du territoire et du public concerné ; le modèle éducatif ayant comme base le binôme échec/réussite doit être abandonné pour laisser la place à des parcours flexibles, permettant la 
capitalisation des compétences acquises et la possibilité d'une réorientation tout au long de la vie scolaire ${ }^{1}$.

\section{L'autonomie des établissements scolaires}

La première disposition prise par le pouvoir institutionnel pour atteindre les objectifs de la réforme a été de doter les établissements scolaires de l'autonomie en matière de didactique et d'organisation, conformément à l'article 21 de la loi Bassanini 59/97 et au décret présidentiel 275/99 2. Selon le propos de l'ancien ministre Berlinguer, l'autonomie est " la mère de toutes les réformes " et elle dessine le cadre à l'intérieur duquel les plus récentes dispositions législatives en matière d'éducation trouvent leur raison d'être ${ }^{3}$.

L'autonomie répond d'abord à la nécessité, qui caractérise l'ensemble des pays de l'Union, de décentralisation du pouvoir, de glissement du dirigisme vertical vers le partage de responsabilités entre l'autorité centrale et le gouvernement du territoire. La notion de prise de décision conjointe est donc le moteur de l'autonomie des établissements scolaires; le défi est de transformer la structure rigide du système éducatif, où les écoles ne représentaient que le dernier maillon d'une chaîne hiérarchique, en un réseau d'institutions autonomes en rapport étroit avec un lieu de coordination au niveau régional assurant l'intégration école-formation-travail.

Cela ne veut pas dire que le centre, représenté par le ministère de l'Instruction publique, abandonne les écoles aux spontanéismes locaux, mais qu'il se transforme en organe d'orientation et de gestion spécifiant les objectifs éducatifs généraux et nationaux, tout en laissant aux écoles la possibilité de choisir librement les parcours à mettre en œuvre pour atteindre ces objectifs.

1 La première nécessité est de faire obtenir un diplôme secondaire, ou mieux, universitaire, à ceux qui restent encore exclus du système éducatif ou qui ne réussissent pas à terminer leurs études, faute d'orientation en cours d'études. Quelques chiffres peuvent montrer la gravité du problème. En Italie, $60 \%$ de personnes n'ont pas de diplôme secondaire contre une moyenne de $40 \%$ en Europe. Seulement 10 \% des Italiens obtiennent un diplôme universitaire, contre $20 \%$ des étudiants européens. Par ailleurs, et c'est alarmant, seulement $38 \%$ des étudiants inscrits à l'université réussissent à achever leur parcours d'étude, alors qu'au Royaume-Uni le taux est de $81 \%$, en Allemagne de $72 \%$, en France de 55 \% (source : Fondazione Giovanni Agnelli sur les données MURST et OECD Education Database, 1999).

2 La loi Bassanini redéfinit les rapports entre le centre et le pouvoir local dans tous les secteurs, y compris celui de l'éducation. Elle établit à l'article 21, alinéas 8 et 9 que les établissements scolaires peuvent, d'une part, diversifier et rendre flexible l'horaire annuel dans la limite de 200 jours de cours fixés par la loi et, d'autre part, choisir la méthodologie et les instruments pour atteindre les objectifs préétablis, y compris la possibilité d'introduire des enseignements facultatifs, optionnels ou additionnels. Le décret présidentiel 275/99 concerne le règlement sur l'autonomie des institutions scolaires qui est entré en vigueur le $1^{\text {er }}$ septembre 2000.

3 D'après l'intervention du ministre Berlinguer, le $1^{\text {er }}$ mars 2000, lors des journées de l'autonomie. 
Cette liberté dépend néanmoins d'une sensibilité que chaque institution scolaire doit avoir à l'égard de la spécificité de la réalité environnante et du public cible de l'offre de formation; elle repose sur l'identification d'un curriculum local qui intègre et relaie celui qui est établi par l'administration centrale, mais respecte les diversités géographiques du pays et les différences culturelles des publics cibles.

Après deux ans d'expérimentations ${ }^{4}$, qui ont servi aux écoles de rodage pour se préparer à cette innovation institutionnelle, le règlement de l'autonomie est entré en vigueur: le $1^{\text {er }}$ septembre 2000, les cloches des écoles italiennes avaient toutes un son différent.

L'une des caractéristiques fondamentales de l'autonomie est d'abord la flexibilité, qui ouvre les écoles sur le territoire, dans l'optique d'un système formatif intégré et, en même temps, les sensibilise à la nécessité de diversifier les parcours et les activités didactiques dans le respect du style d'apprentissage de chaque élève.

La flexibilité permet d'abord de considérer le système de formation et d'instruction comme un tout complexe permettant l'interaction entre le parcours éducatif de l'école, la formation professionnelle et les expériences de travail : un système de passerelles entre les filières du secondaire est mis en place, des parcours intégrés d'instruction et de formation ou des périodes de stage en entreprise sont prévus. Tout doit donc concourir à la formation de l'élève et à la capitalisation des expériences acquises, dans le but de faire coïncider le parcours formatif et le projet de vie personnel et de garantir à chacun une formation réussie ${ }^{5}$.

Chaque école doit prévoir, en outre, une flexibilité interne qui permette d'articuler le rapport enseignement/apprentissage d'une manière souple et adaptable aux exigences de chaque élève. Parmi les formes de flexibilité prévues

4 Dès l'année scolaire 1998/1999, les financements de la loi 440/97 ont permis aux écoles de commencer un large travail d'innovation et d'introduction de quelques éléments de flexibilité concernant la future autonomie. L'année suivante, un nouveau programme d'expérimentation a proposé aux écoles un travail d'innovation plus cohérent, fondé sur l'élaboration du plan de l'offre de formation (POF) dans lequel est précisé le projet formatif adopté par chaque établissement scolaire. Dans le cadre de l'expérimentation, des projets spéciaux (Lingue 2000, bibliothèques, musique...) ont été mis en place, également financés par les fonds de la loi 440/97, dans le but d'accompagner la réforme et de soutenir l'école de l'autonomie. Le projet Lingue 2000, par exemple, propose un curriculum de langues vivantes en continuité de la maternelle à la terminale et tente de réorganiser le parcours formatif en termes d'objectifs d'apprentissage et de compétences à atteindre, capitaliser et certifier. Pour les détails concernant les innovations dans le secteur des langues, voir aussi, dans la deuxième partie de cet article, le chapitre consacré à la valorisation des langues vivantes et des TIC.

5 Voir articles 4 et 8 du DPR 275/99 sur l'autonomie des institutions scolaires. La hausse de la limite d'âge de l'obligation scolaire à 15 ans (loi 9/99) et l'introduction de l'obligation de formation à 18 ans (loi 144/99) sont à situer dans la perspective de l'autonomie et donnent naissance, grâce aussi à la loi 167/97 réglementant la formation professionnelle et le stage en entreprise, à un véritable système formatif intégré. 
par le règlement, on peut citer : l'articulation modulaire ${ }^{6}$ de l'horaire annuel de chaque discipline ou activité, une réduction horaire de l'unité d'enseignement et l'utilisation plus souple du temps restant par des parcours intégratifs, la mise en place de parcours didactiques individualisés, l'élargissement de l'offre de formation par des cours/disciplines supplémentaires, l'articulation flexible du groupe-classe et sa subdivision en sous-groupes venant aussi de classes différentes pour des moments de soutien, rattrapage ou approfondissement, l'agrégation des disciplines en aires disciplinaires plus vastes et la possibilité d'effectuer des compensations entre les disciplines et les activités curriculaires. À tout cela, il faut ajouter la flexibilité concernant l'horaire d'enseignement qui permet de prévoir des parcours pour l'accueil, l'orientation et la réorientation (système des passerelles), des formules d'enseignement intensives suivies de périodes de simple maintien des compétences, la diminution enfin du nombre des disciplines par la concentration de l'horaire de certains cours sur des périodes plus courtes (3 ou 4 mois).

La flexibilité de l'activité éducative est explicitée dans le plan de l'offre de formation (piano dell'offerta formativa, POF), carte d'identité de chaque école, établi par le conseil des enseignants et adopté par le conseil d'établissement, dans lequel sont aussi précisés les cours de soutien pour les élèves en difficulté, les activités de recherche et d'expérimentation, les accords passés avec d'autres écoles, qui peuvent également comprendre des échanges d'enseignants. D'après le règlement, le $\mathrm{POF}$ est « le document fondamental constitutif de l'identité culturelle et du projet des établissements scolaires. Il est présenté aux élèves et à leurs parents lors de l'inscription à l'école, et décrit la réalité de l'établissement tout en répondant aux exigences locales et du public concerné. " ${ }^{7}$ Le POF n'est pas un modèle rigide, puisqu'il doit s'adapter au contexte culturel, social, et économique de son environnement, mais l'équipe de coordination nationale de l'autonomie, opérant auprès du ministère de l'Instruction publique, a fourni un schéma d'ensemble indicatif, qui peut aider les établissements scolaires à élaborer leur projet éducatif (voir tableau 1).

\section{La décentralisation des structures éducatives}

Pour que ce nouveau mécanisme de décentralisation des compétences en matière d'instruction et de formation puisse produire les effets de délocali-

6 Par le terme " module " on entend, d'une façon synthétique, un segment ou une unité d'apprentissage disciplinaire ou pluridisciplinaire possédant une cohésion et une cohérence thématique et permettant d'acquérir des compétences spécifiques, vérifiables et capitalisables en tant que crédits de formation.

7 Voir l'article 2 du DPR 275/99 sur l'autonomie. 


\section{Tableau 1 \\ Le Plan de l'offre de formation}

\section{Conditions générales du service scolaire}

- structures et services de l'établissement

- règlements et statuts de l'école : droits et devoirs, modalités de participation

- horaires d'ouverture

- structures et services que les organismes locaux ou d'autres entités mettent à la disposition de l'école

- relations avec le tissu urbain

\section{Cursus proposés}

- traditionnels

- expérimentations

- horaire annuel obligatoire

- disciplines de la quote-part du curriculum national

- compétences acquises en sortie

Curricula : parcours conçus par chaque institution scolaire pouvant être individualisés à travers des mesures de flexibilité

- disciplines et activités de la quote-part du curriculum décidées par l'école

- compensation entre les disciplines

- options offertes aux élèves et aux familles

- disciplines et activités supplémentaires

- recherche et expérimentation

- modalités et critères d'évaluation des élèves, validation des crédits

- actions

- accueil

- continuité

- orientation et réorientation (système de passerelles)

- aide personnalisée et soutien (modules de allineamento/ajustement ${ }^{*}$ )

- encouragement des excellences

- organisation du temps

- adaptation du calendrier scolaire

- articulation de l'horaire annuel de chaque discipline et des activités

- utilisation de $15 \%$ de l'horaire annuel pour permettre d'éventuelles compensations entre les disciplines

- modalités d'adaptation des horaires d'enseignement au rythme d'apprentissage de chaque élève

"Les moduli di allineamento s'adressent à de petits groupes organisés en parallèle sur plusieurs classes, où les élèves sont aidés dans un travail individuel de rattrapage tout en suivant en même temps le programme disciplinaire ordinaire. 
- lieux

- classes

- laboratoires

- gymnase

- extérieur (théâtres, universités, voyages éducatifs...)

- groupes

- classes

- groupes flexibles d'élèves venant de la même classe ou de classes différentes

- groupes d'élèves fréquentant des disciplines ou des activités optionnelles/supplémentaires

\section{Relations}

- mise en réseau avec d'autres établissements

- relations avec le territoire local

\section{Ressources humaines}

- modalités d'utilisation du personnel éducatif et administratif

- modalités d'organisation de l'établissement

\section{Ressources financières synthétisées dans un devis contenant}

- financement des projets

- financement des activités supplémentaires d'enseignement (pour les enseignants de l'établissement et les intervenants extérieurs)

- financement des activités supplémentaires hors enseignement (pour les enseignants de l'établissement et le personnel administratif)

- frais de transport ou d'autres services

- frais d'achat de matériel

- frais découlant des conventions ou des accords en réseaux

- frais de formation du personnel éducatif et administratif

- divers

Critères pour le suivi, l'évaluation des résultats et les actions d'ajustement qui en découlent

- indicateurs

- public et personnel concernés

- éventuels observateurs externes

- calendrier de réalisation

- actions d'adaptation et d'ajustement

- documentation

\section{Projets conçus comme expression cohérente de l'établissement}

- actions (définition de la problématique et description du projet)

- phases et modalités de la mise en œuvre du projet (calendrier, lieux, groupes...)

- ressources financières

- définition des indicateurs de succès pour la mise en œuvre du suivi 
sation voulus, tout en préservant l'unité du système éducatif, le législateur devait s'engager en même temps à mettre en place une structure administrative cohérente. Le décret législatif 300/99 sur le réaménagement des ministères et le règlement sur l'organisation du ministère de l'Instruction publique, approuvé le 24 juillet par le Conseil des ministres, définissent les différentes aires de compétence, l'articulation interne du pouvoir central ainsi que le nouveau rôle de l'administration territoriale.

La structure ministérielle cesse d'avoir une vocation principalement hiérarchique pour exercer notamment des fonctions d'orientation, de coordination et de programmation du système d'instruction, à travers l'institution de deux départements et de trois services autonomes:

- le département pour le développement de l'instruction s'articule à son tour en trois aires de compétence : le système scolaire et les programmes, y compris les examens, les diplômes et les certifications, la recherche et l'expérimentation des innovations; la formation et le recyclage du personnel éducatif; les relations internationales et la coopération éducative dans le cadre de l'Union européenne ;

- le second département pour les services sur le territoire prévoit, lui aussi, des aires distinctes : l'organisation des services territoriaux, y compris les services pour l'intégration et l'accueil ; l'instruction supérieure et l'éducation tout au long de la vie, les parcours intégrés d'instruction et de formation professionnelle; la gestion du personnel éducatif et de l'administration; les politiques de la jeunesse et les rapports école-activités sportives;

- trois services indépendants de niveau directionnel sont mis en place pour assumer des fonctions d'intérêt commun aux départements et à l'administration régionale. Ils s'occupent d'affaires économiques et financières, d'informatisation et d'innovation technologique, de communication institutionnelle notamment par le biais de l'intranet et du multimédia.

Au niveau régional, les Sovrintendenze scolastiche regionali (rectorats/ académies au niveau régional) et les Provveditorati agli Studi (au niveau provincial) sont remplacés par un centre organisationnel dans les chefs-lieux de chaque région avec des pouvoirs spécifiques, notamment sur le budget. Les bureaux scolaires régionaux mettent en œuvre les dispositions générales en matière d'instruction et exercent plusieurs fonctions : le soutien aux établissements scolaires autonomes; les relations avec les administrations régionales, les organismes locaux, les universités et les agences de formation; le recrutement et la mobilité du personnel éducatif; la gestion des ressources financières et du personnel des écoles. Enfin, des services de conseil et de soutien aux établissements scolaires autonomes sont pareillement mis en place au niveau provincial. 


\section{La réorganisation des cycles scolaires et les travaux de la commission des Sages}

La réorganisation des cycles scolaires, conformément à la loi 30/2000 ${ }^{8}$, devient l'élément le plus significatif de la réforme envisagée par l'ancien ministre Berlinguer et en représente le noyau fondamental. Dans l'école de l'avenir, comme on l'a vu dans le règlement sur l'autonomie, un rôle décisif sera joué par le système d'orientation et d'intégration entre l'instruction et la formation professionnelle, dans le but d'offrir aux jeunes un parcours en continuité, de l'école jusqu'à l'université, la formation intégrée supérieure et le monde du travail. La réduction du nombre des cycles, de trois (cycles élémentaire, moyen, secondaire) à deux (de base et secondaire) vise à respecter les deux phases fondamentales du processus d'apprentissage (voir tableau 2 et tableau 3 ).

Tableau 2

Le système actuel

\begin{tabular}{|c|c|c|c|c|c|c|}
\hline $\begin{array}{c}\text { École } \\
\text { maternelle }\end{array}$ & $\begin{array}{c}\text { École } \\
\text { élémentaire }\end{array}$ & & $\begin{array}{c}\text { École } \\
\text { moyenne }\end{array}$ & & $\begin{array}{c}\text { École } \\
\text { secondaire }\end{array}$ & $\begin{array}{c}\text { Diplôme d'État } \\
\text { à } 19 \text { ans }\end{array}$ \\
\hline $\begin{array}{c}\text { durée : } \\
3 \text { années } \\
\text { (âge : } 3-5 \text { ans) }\end{array}$ & $\begin{array}{c}\text { durée: } \\
5 \text { années } \\
\text { (âge : } 6-11 \text { ans) }\end{array}$ & Examen & $\begin{array}{c}\text { durée: } \\
3 \text { années } \\
\text { (âge }: 12-14 \text { ans) }\end{array}$ & Examen & $\begin{array}{c}\text { durée : } \\
5 \text { années } \\
\text { (âge }: 15-19 \text { ans) }\end{array}$ & \\
\hline
\end{tabular}

Tableau 3

Le système futur

\begin{tabular}{|c|c|c|c|c|c|c|}
\hline \multirow{2}{*}{\begin{tabular}{|c|}
$\begin{array}{c}\text { École } \\
\text { de l'enfance }\end{array}$ \\
durée : \\
3 années \\
(âge $: 3-5$ ans)
\end{tabular}} & \multirow{2}{*}{$\begin{array}{c}\begin{array}{c}\text { École } \\
\text { de base }\end{array} \\
\text { durée : } \\
7 \text { années } \\
\text { (âge : } 6-13 \text { ans) }\end{array}$} & \multirow[b]{2}{*}{ Examen } & \multicolumn{3}{|c|}{ Cycle secondaire } & \multirow{2}{*}{$\begin{array}{c}\text { Diplôme d'État } \\
\text { à } 18 \text { ans }\end{array}$} \\
\hline & & & $\begin{array}{c}2 \text { années } \\
\text { d'orientation } \\
\text { (âge : } 14-15 \text { ans) }\end{array}$ & $\begin{array}{c}\text { Fin scolarité } \\
\text { obligatoire }\end{array}$ & $\begin{array}{c}3 \text { années } \\
\text { de spécialisation } \\
\text { (âge }: 16-18 \text { ans) }\end{array}$ & \\
\hline
\end{tabular}

Dans la structure envisagée, l'école de base d'une durée de sept années garantira un parcours éducatif unitaire articulé selon les exigences de l'évolution des élèves, en vue de les conduire à une maturité suffisante pour l'entrée dans le cycle suivant. Le cycle secondaire, de cinq années, s'articulera en plusieurs aires : classico-humaniste, scientifique et technologique, artistique et musicale. Les écoles auront toutes le nom de lycées, ce qui mettra fin, par consé-

8 Loi du 10 février 2000, $n^{\circ}$ 30, sur la réorganisation des cycles de l'instruction, publiée dans la Gazzetta Ufficiale, $n^{\circ} 44,23$ février 2000. 
quent, à la séparation nette entre les cursus plus généraux et ceux qui sont orientés vers la profession. La nouvelle structure, tout en réduisant considérablement le nombre actuel de filières, permettra une orientation préalable et le passage graduel au dernier segment de spécialité. Les deux premières années de ce cycle qui concluent le segment d'obligation scolaire mettront en place, conformément à ce que le règlement sur l'autonomie établit, un système de passerelles permettant le passage d'un cursus à un autre et vers d'autres parcours de formation. Le diplôme final d'État sera délivré après douze années de scolarité - dont neuf de scolarité obligatoire et trois d'obligation de formation - en harmonie avec les autres pays européens.

Si les trois cycles scolaires encore en vigueur ne prévoient aucune période d'orientation, en revanche, le système à venir prendra en compte les difficultés des jeunes qui auront fait, à partir de représentations erronées, des choix hâtifs de filières, et leur permettra plus facilement de modifier leur parcours dans le système formel d'instruction ${ }^{9}$.

\section{Les travaux de la commission des Sages}

Pour passer du cadre normatif de référence à la mise en œuvre graduelle de la réorganisation des cycles, le ministre Tullio de Mauro a constitué une commission de 280 experts, divisée en neuf groupes de travail. Le rapport qui vient d'être rédigé, accompagné d'un document opérationnel en cours de préparation, permettra au gouvernement de soumettre au parlement un programme articulé sur cinq ans ${ }^{10}$.

Les priorités indiquées dans le document sont: l'institution d'un professeur senior assumant la tâche de tuteur et coordonnateur des professeurs

9 À ce propos, il convient de citer l'article 7 de la loi 30/00 qui prévoit au paragraphe 7 : « La fréquentation positive de tout segment de l'école secondaire, annuel ou modulaire, comporte l'acquisition d'un crédit de formation valable pour reprendre les études interrompues, pour passer d'une aire ou d'une filière à une autre ou à la formation professionnelle. De la même manière, la fréquentation positive de segments de formation professionnelle comporte l'acquisition de crédits valables pour accéder au système d'instruction. " I nous semble aussi utile de citer l'article 6 (passage entre les systèmes) du règlement 257/00 sur l'obligation de fréquentation des activités de formation jusqu'à 18 ans : "Les connaissances, les compétences et les savoir-faire acquis dans le système de formation professionnelle, dans les stages en entreprise, pour effet de l'activité de travail ou en autoformation, constituent des crédits pour l'accès aux différentes années de l'école secondaire. ”

10 Le plan du gouvernement devra prévoir les critères généraux pour la réorganisation des curricula de l'école de l'enfance, de base et secondaire, y compris ceux qui portent sur la valorisation des langues vivantes et des technologies ; le projet de requalification du personnel éducatif ; les critères généraux pour la dotation de personnel dans les établissements scolaires ; le plan de réaménagement des infrastructures ; les conditions de faisabilité de la mise en œuvre de la réforme et de l'adaptation des moyens à disposition par rapport aux objectifs ; l'évaluation de l'éventuelle majoration ou diminution des frais à engager. 
plus jeunes ; l'articulation flexible du groupe-classe au cours de l'année scolaire, selon les matières et les activités; la révision des disciplines et la réorganisation des curricula ainsi que la diminution des filières.

En ce qui concerne les objectifs généraux de la réforme, les experts signalent la nécessité de dépasser la "rigidité d'un système centralisé de gestion de l'école axé sur une réglementation démesurée empêchant la qualité de l'apprentissage ». De plus, il est nécessaire que les établissements ne soient pas isolés dans leur innovation et que le nouvel aménagement territorial permette l'interaction avec les entités et organismes locaux, pas seulement au niveau régional. Il faut, en effet, éviter une nouvelle forme de dirigisme central, certes de dimension plus réduite, qui risquerait de déresponsabiliser les écoles.

La réforme des cycles, d'après le document final, doit conduire à la définition d'une culture du travail; il en découle le nouveau rôle à attribuer à la formation professionnelle et aux deux premières années d'orientation du secondaire qui concluent la scolarité obligatoire.

En ce qui concerne l'organisation horaire, les experts ont proposé certaines orientations, notamment pour l'articulation curriculum national/curriculum local, qui ont été reprises dans de récentes décisions officielles ${ }^{11}$. Pour le premier cycle de sept ans, le volume horaire sera autour de 1000 heures par an, avec une quote-part locale de $25 \%$ déterminée par chaque établissement scolaire. Le cycle secondaire comportera aussi un volume horaire de 1000 heures environ par an, mais le curriculum local atteindra $20 \%$ du montant horaire d'ensemble. Cette quote-part autonome pourra augmenter jusqu'à $30 \%$ dans les deux premières années pour prévoir des activités de rattrapage et d'orientation et jusqu'à $40 \%$ dans les trois années de spécialisation pour garantir dans les différentes filières des enseignements optionnels, dont la liste sera établie au niveau national.

\section{La valorisation des langues et des TIC}

Le rapport de la commission développe également deux des principaux thèmes sur lesquels se focalisent l'évolution du système éducatif italien et le caractère novateur de la réforme en acte: l'enseignement/apprentissage des langues vivantes et l'utilisation des technologies de l'information et de la communication. Le groupe de travail 4 a défini, à partir des expérimentations conduites en la matière, des principes pour le développement des curricula et la définition des référentiels essentiels de compétence.

Les principes généraux suggérés dans le document de synthèse sont d'abord ceux de la verticalité et de la continuité ; cela demande la redéfinition

11 Programme du gouvernement pour cinq ans, présenté le 17 novembre 2000, et résolution de la Chambre des députés du 12 décembre 2000. 
des parcours didactiques en termes d'objectifs d'apprentissage et de compétences à certifier, de manière à permettre le passage d'un cycle à un autre ou d'une filière à une autre en évitant les répétitions et, par conséquent, la démotivation des élèves.

Pour ce qui concerne les langues, la transformation semble accélérée et facilitée par l'expérimentation, lancée en 1999, du projet Lingue 2000, qui s'insère dans le cadre des politiques éducatives européennes et se réfère aux documents élaborés par le Conseil de l'Europe ${ }^{12}$; son but est de faire atteindre des compétences communicatives transparentes et certifiables dans au moins deux langues vivantes.

Le projet propose un parcours en continuité, garantissant l'apprentissage en groupes réduits d'élèves (12 à 15), parfois même grâce à des cours extracurriculaires que chaque école peut intégrer dans son POF d'après les exigences et les demandes de son public. Le volume horaire est réparti sur les différents cycles (maternelle, élémentaire, moyen, secondaire) et l'horaire annuel se subdivise en brefs modules ou unités d'apprentissage (de 20 à 30 heures environ) contenant les objectifs spécifiques et les contenus sous forme de descripteurs des compétences à atteindre. Cette organisation permet aux élèves d'obtenir le cumul de crédits tout au long du parcours d'apprentissage. Les niveaux de compétence à atteindre à la sortie de chaque segment scolaire répondent aux descripteurs de niveau contenus dans le Cadre commun européen de référence du Conseil de l'Europe. Il est également possible dans ce contexte de demander une validation externe des niveaux atteints, effectuée par des organismes de certification reconnus et agréés au niveau international, ce qui répond aussi à l'exigence d'ouverture, de la part des écoles, aux offres de formation et de service de qualité venant de l'extérieur ${ }^{13}$.

Les experts du groupe sont donc partis de la conviction qu'il fallait valoriser les grands axes de l'innovation du projet Lingue 2000, y compris la volonté d'anticiper l'enseignement/apprentissage des langues dès le plus jeune âge, et l'adapter au fur et à mesure à l'évolution et aux besoins des apprenants.

12 Les documents du Conseil de l'Europe auxquels on fait ici référence sont le Cadre commun européen de référence (Projet 2, Strasbourg, 1997) et le Portfolio européen des langues (Strasbourg 1997). Le premier, en particulier, trace un cadre de référence qui détermine la langue que l'apprenant doit être capable de pratiquer pour communiquer avec succès à différents niveaux de compétence (du niveau débutant à la maîtrise), les compétences et les stratégies qu'il doit activer, les méthodes qu'il peut utiliser pour apprendre ou acquérir telle compétence, les types d'évaluations intermédiaires ou finales qui peuvent témoigner des niveaux atteints.

13 Chaque établissement scolaire peut donc ajouter à la valeur d'une évaluation interne celle d'une certification externe, valable aussi dans le monde du travail et hors d'Italie. La certification des compétences en sortie de chaque segment peut être délivrée par des organismes certificateurs reconnus et agréés pour les quatre langues les plus diffusées (anglais, français, espagnol et allemand) qui ont signé un protocole d'accord avec le ministère italien de l'Instruction publique. 
En ce qui concerne les technologies de l'information et de la communication, à part les indicateurs utilisés pour l'obtention du permis de conduire informatique européen, de véritables référentiels au niveau plus conceptuel ne sont pas disponibles à l'heure actuelle. Les experts souhaitent donc faire référence à ceux que l'on utilise dans d'autres pays. Les TIC sont, en outre, considérées dans le document de synthèse comme des instruments essentiels pour l'introduction de modèles innovants d'apprentissage et d'organisation de la didactique, qui permettent de mettre l'accent plus sur l'élaboration que sur la transmission des connaissances.

Comme pour les langues vivantes, l'enseignement informatique devrait commencer à l'école de l'enfance et se relier notamment à des activités ludiques pour continuer jusqu'à la terminale, même à travers des intégrations extracurriculaires ou en tant que discipline de la quote-part locale. Dans les deux premières années du secondaire, en outre, on conseille d'introduire les TIC en tant que véritable discipline faisant partie du curriculum national, afin d'approfondir ses principes constitutifs et de les intégrer dans l'ensemble des connaissances à atteindre avant l'issue de la scolarité obligatoire. Tout cela demande forcément un niveau satisfaisant de structures et d'équipement pour chaque établissement scolaire, la nomination d'un responsable du système informatique dans chaque école et la mise en réseau des établissements, permettant l'échange, la documentation et la diffusion des bonnes pratiques ${ }^{14}$.

\section{La requalification du personnel enseignant}

Les enseignants représentent, pour la qualité d'un système scolaire intégralement rénové, une ressource stratégique. Il n'existera pas d'école nouvelle sans que soient prévues une requalification et une mise en valeur du personnel éducatif qui doit assumer des tâches plus lourdes et de nouvelles responsabilités.

Pour cela il est nécessaire, d'après le rapport des Sages, de mettre en place des activités significatives de formation continue, y compris des parcours spécifiques d'enrichissement professionnel et un système de reconnaissance de crédits (professionnels, culturels, concernant l'organisation scolaire). Il est, en outre, souhaitable de faire bénéficier les professeurs de périodes sabbatiques

14 En ce qui concerne la nécessité que les écoles s'équipent au niveau informatique, il est utile de rappeler le programme, sur trois ans, de développement des technologies didactiques (PSTD), lancé par le ministère de I'Instruction publique en 1997. II s'agit d'un programme à grande échelle impliquant tout le système scolaire italien et incluant la dotation directe aux écoles de financements pour l'acquisition de matériel, de logiciels et pour la formation des professeurs. Le programme se divise en deux volets : le premier destiné aux écoles avec peu d'expérience ou sans expérience ; le second destiné à équiper une ou plusieurs classes afin que le multimédia soit concrètement intégré à la didactique. D'une façon générale, les écoles entrent d'abord dans le premier et, ensuite, dans le second. L'un des objectifs principaux du programme est la mise en réseau des établissements sur internet. 
pour le recyclage et de stages de spécialisation, même à l'étranger. Les experts envisagent également la création du profil professionnel de professeur-chercheur, un professeur jouissant d'une rétribution additionnelle avec une qualification de niveau plus élevé que la simple formation initiale du professeur de base. Par ailleurs, le groupe de travail qui s'est penché sur la promotion professionnelle des enseignants demande au gouvernement d'établir dans le détail l'expertise et la compétence que les futurs professeurs devront posséder; leur préparation devrait prévoir une formation aussi bien disciplinaire que pédagogique et didactique. On ne peut pas enseigner une discipline si on ne connaît pas l'élève, on n'enseigne pas à l'élève - même si on sait tout de lui - si on ne connaît pas la discipline.

Le groupe a aussi examiné la question assez délicate des éventuelles modalités d'évaluation des enseignants. Une des suggestions proposées est de recourir à un organisme déjà existant dans les écoles, le comité d'évaluation du service, qui reste en réalité largement inutilisé. Il s'agit à l'heure actuelle d'un groupe constitué par le chef d'établissement et deux ou quatre enseignants, chargé d'évaluer le travail et la compétence professionnelle, exclusivement sur demande d'un professeur de l'établissement. En fait, ce comité ne se réunit que pour l'évaluation, obligatoire aux termes de la loi, du professeur néo-titulaire après sa première année de service.

Le groupe des experts, quant à lui, propose que chaque école utilise le comité d'évaluation pour mesurer la compétence professionnelle des enseignants et la lier éventuellement à des rétributions additionnelles ou à des avancements de carrière. Le problème reste encore ouvert, et il n'est pas actuellement possible de dire si la proposition formulée peut être prise en compte par le législateur, et si l'une des questions les plus épineuses peut véritablement être résolue par une simple disposition de loi.

\section{Quelques considérations finales}

Il semble utile de se livrer, en conclusion, à quelques considérations concernant certains des aspects de ces évolutions, plus délicats, et qui suscitent encore des doutes.

\section{La dimension locale et la dimension nationale}

Tout d'abord, l'autonomie est désormais une réalité opérationnelle sur l'ensemble du territoire italien; il est impossible de penser à un retour en arrière et à une prise de décision politique inverse qui reporterait l'école à un état de dirigisme. Il y a néanmoins encore des risques, liés au manque de support véritable au niveau local. La réforme de l'administration n'est pas encore réalisée, sauf pour quatre régions qui ont introduit d'une manière expérimentale les directions régionales en soutien au processus de l'autonomie 
scolaire ${ }^{15}$. Ce manque d'un organisme local de référence risque de dénaturer le rapport école/réalité environnante. Un exemple : pour permettre l'intégration entre les écoles secondaires et la formation professionnelle, nœud central de l'école de l'autonomie ouverte sur le territoire, chaque école devrait pouvoir s'adresser à un organisme régional offrant une liste des cours et des agences de formation.

Ensuite, comme on l'a déjà précisé dans la première partie de cet article, la valorisation de la dimension locale nécessite parallèlement de définir au niveau central les compétences, les référentiels et les objectifs disciplinaires et transversaux qui garantissent un cadre unitaire au système de formation et font dès lors de la diversité locale une richesse nationale.

Des mesures de péréquation devraient enfin être envisagées pour permettre aux écoles, dans le cas où les ressources territoriales feraient défaut, de développer et de gérer au mieux leur projet d'établissement. Un tel correctif, accompagné d'un contrôle au niveau national, éviterait de créer un système éducatif à deux vitesses: les zones riches avec leur tradition de formation de qualité, progressant rapidement vers le succès, et les zones désavantagées qui s'effondrent faute de mesures appropriées.

\section{Les ressources financières et humaines}

Comme le rapport final de la commission des experts le souligne, une autre question à résoudre pour éviter la déception des opérateurs éducatifs et du public concerne la définition des ressources. Il faut d'abord redéfinir d'une façon plus souple l'organisation du travail des enseignants et doter les écoles de ressources humaines suffisamment efficientes et capables de collaborer à la gestion du projet éducatif global.

Ensuite, la flexibilité de l'autonomie demande des investissements en termes de structures scolaires, de manière à permettre la division du groupeclasse, la constitution de centres de ressources équipés et pouvant répondre à la demande de documentation, la mise en réseau entre établissements et avec les organismes de formation du territoire.

Enfin, il faut introduire un système budgétaire qui, à côté de celui qui permet le financement au coup par coup, limite les obligations de destination tout en autorisant les transferts d'une ligne budgétaire à une autre.

\section{Le suivi et l'évaluation}

Il conviendra, pour suivre et contrôler le processus de réforme, de mettre en place un suivi à deux niveaux : d'une part, une observation locale

15 Depuis mai 2000, les bureaux scolaires régionaux de Toscane, de Lombardie, de Ligurie et de Sicile ont été institués sous forme expérimentale. 
permettant la gestion raisonnée des innovations éducatives, pour envisager les éventuels correctifs à apporter en cours de réalisation et pour diffuser les bonnes pratiques; d'autre part, un suivi national prenant en compte le processus d'ensemble ${ }^{16}$.

La mesure des performances des élèves et le contrôle de qualité du service offert par les établissements représentent sans aucun doute l'un des nœuds essentiels de la réforme en acte. Un premier pas a été fait le 15 septembre, avec la définition par le Conseil des ministres du règlement de l'Institut national pour l'évaluation, ex-CEDE (Centre européen pour l'éducation) ${ }^{17}$. La nouvelle structure devra élaborer et soumettre périodiquement aux élèves un test pour évaluer leur niveau d'apprentissage. Dans l'avenir, l'institut devra aussi mettre sur pied un classement des écoles en fonction de la qualité de leur service.

\section{**}

Les différents points évoqués constituent les aspects les plus significatifs de la réorganisation des cycles et de l'introduction de l'autonomie. L'objectif est ambitieux, l'enjeu considérable, et il est presque certain qu'il faudra apporter des ajustements en cours de réalisation.

C'est maintenant à l'école et à la société civile d'accepter le défi et de permettre la mise en œuvre de cette vaste réforme, qui répond à la fois à des changements de perspective quant à l'enseignement/apprentissage, et à la conviction de plus en plus partagée que l'amélioration du « système-pays " passe aussi par l'aménagement d'un système éducatif de qualité.

\section{Sites}

www.istruzione.it, le site officiel du ministère de l'Instruction publique sur lequel on trouve les lois, les règlements, les décrets cités, concernant l'instruction et la formation en général.

www.istruzione.it/argomenti/autonomia/default.htm, voir les pages dédiées à l'autonomie et aux projets spéciaux.

http://culture.coe.fr/lang/indexfr.html, les documents du Conseil de l'Europe.

16 Pendant la phase expérimentale de l'autonomie et des projets spéciaux (1998-2000), un suivi a accompagné et mis en lumière les aspects innovants du processus réformateur et la réaction des écoles à ce processus. 17 Le règlement concernant l'Institut national pour l'évaluation du système d'instruction permet la mise en œuvre des articles 1 et 3 du décret législatif 258 du 30 juillet 1999, qui avait établi la transformation du CEDE. En résumé, les devoirs et les tâches du nouvel institut seront: l'évaluation de l'efficacité du système éducatif ; l'étude des causes de l'abandon scolaire ; l'évaluation des effets des dispositions législatives ; la diffusion de la culture de l'évaluation et de l'auto-évaluation ; l'évaluation des projets mis en place à l'échelle nationale. 